Matsumoto et al. Ocular blood flow levels with CRVO after IVB.

\title{
Retinal blood flow levels measured by Laser Speckle Flowgraphy in patients who received intravitreal bevacizumab injection for macular edema secondary to central retinal vein occlusion
}

\author{
Abbreviated title: Retinal blood flow with CRVO after IVB \\ Makiko Matsumoto, MD, PhD; Kiyoshi Suzuma, MD, PhD; Yoshiko Fukazawa, \\ MD; Yoshihisa Yamada, MD, PhD; Eiko Tsuiki, MD, PhD; Azusa Fujikawa, MD, \\ PhD; and Takashi Kitaoka, MD, PhD
}

Department of Ophthalmology and Visual Sciences, Graduate School of Biomedical Sciences, Nagasaki University, Nagasaki, Japan

Correspondence to: Kiyoshi Suzuma, MD, PhD

Department of Ophthalmology and Visual Sciences, Graduate School of Biomedical Sciences, Nagasaki University, 1-7-1 Sakamoto, Nagasaki 852-8501, Japan

Fax: 011-81-95-819-7347; Tel: 011-81-95-819-7345

E-mail: suzuma@nagasaki-u.ac.jp

There were no grants or any external funding sources used to support this research.

The authors have no proprietary or conflicts of interest for this study. 
Matsumoto et al. Ocular blood flow levels with CRVO after IVB.

Key Words: bevacizumab, blood flow, central retinal vein occlusion, Laser Speckle Flowgraphy

Summary statement: Retinal blood flow levels in CRVO patients after IVB.

\begin{abstract}
Purpose. To report retinal blood flow levels measured by Laser Speckle Flowgraphy (LSFG) in three patients after they received an intravitreal bevacizumab injection (IVB) for macular edema secondary to central retinal vein occlusion (CRVO).

Methods. Three patients (3 eyes) being treated with IVB $(1.25 \mathrm{mg} / 0.05 \mathrm{ml})$ for secondary macular edema of CRVO were examined. LSFG analyses of the blood flow were based on examinations of the mean blur rate (MBR) at the major vessels of the optic disc. Central retinal thickness (CRT) was measured by optical coherence tomography (OCT) using Macular Cube $512 \times 128$ scanning protocol.
\end{abstract}

Results. After the first IVB, case 1 exhibited an increase in MBR and a decrease in CRT. Due to a subsequent MBR decrease and CRT increase four months later, an additional injection was required, which led to an increase in MBR and a decrease in CRT, similar to that observed after the first treatment. Subsequently, blood flow has continued to improve without additional IVB. Macular edema recurrence in case 2 led to three further IVBs over a six-month period. Although increases in MBR and decreases in CRT were noted, MBR values tended to decline after each IVB. In case 3, macular edema recurrence led to five 
Matsumoto et al. Ocular blood flow levels with CRVO after IVB.

additional IVBs being carried out within a one-year period. Continuous MBR increases and CRT decreases were observed in the patient after each IVB. By measuring MBR using LSFG, we may predict the prognosis of CRVO.

Conclusions. MBR increases after IVB were confirmed by LSFG in three patients. Even though CRVO pathology backgrounds can vary, LSFG may be useful in both determining the CRVO prognosis and in evaluating treatment efficacy.

\section{Precis.}

MBR increases after IVB were confirmed by LSFG in three patients. Even though CRVO pathology backgrounds can vary, LSFG may be useful in both determining the CRVO prognosis and in evaluating treatment efficacy. 
Matsumoto et al. Ocular blood flow levels with CRVO after IVB.

Vascular endothelial growth factor (VEGF) has been shown to be associated with central retinal vein occlusion (CRVO) pathology. ${ }^{1,2}$ Therefore, anti-VEGF therapy has been proposed as a potential CRVO treatment strategy. CRVO is a disease in which the degree of ischemia varies depending upon both the individual case and the actual stage of the disease. Laser Speckle Flowgraphy (LSFG) (LSFG-Navi, Softcare, Fukuoka, Japan) is a new clinical device that can be conveniently applied to visualize and measure blood flow distribution in the ocular fundus. ${ }^{3,4}$ We are unaware of previous studies that have examined retinal blood flows before and after intravitreal bevacizumab injection (IVB) in CRVO cases. Therefore, this study used LSFG to investigate ocular blood flow levels in three patients after they received IVB for macular edema secondary to CRVO. Methods. Three patients with macular edema due to CRVO were given off-label intravitreal bevacizumab (Avastin; Roche, Reinach, Switzerland). All three patients provided written informed consent prior to treatment initiation. The intravitreal dosage of bevacizumab was $1.25 \mathrm{mg} / 0.05 \mathrm{ml}$. All injections were performed in accordance with standard sterile methodology, ${ }^{5}$ and then followed by administration of prophylactic topical antibiotics at 3 days post injection. To evaluate retinal blood flow levels, mean blur rate (MBR) at the major vessels of the optic disc was determined by LSFG-NAVI, as has been previously described. ${ }^{6}$ LSFG analyzer software (version 3.1.3) was used to calculate MBR in the major vessel area of the optic disc, which was averaged over the vessel area.

Central retinal thickness (CRT) was measured as mean retinal thickness in the central $1000-\mu \mathrm{m}$ diameter area by optical coherence tomography (OCT) 
Matsumoto et al. Ocular blood flow levels with CRVO after IVB.

(CirrusTM HD-OCT, Carl Zeiss Meditec, Dublin, CA) using Macular Cube $512 \times$ 128 scanning protocol.

Case Report 1. A 72-year-old woman noticed a decrease in her right visual acuity and developed macular edema secondary to CRVO. Best-corrected visual acuity was $20 / 70$ in her right eye. A color fundus photograph of the right eye with CRVO showed extensive retinal hemorrhage, slightly tortuous retinal veins, and diffuse retinal edema (Figure 1A). A fluorescein angiogram of the same eye (Figure 1B) demonstrated there was neither a nonperfusion area nor neovascularization. Results from OCT imaging showed CRT of $402 \mu \mathrm{m}$, and MBR was 32.8 (Figure 2A). The patient's macular edema that was secondary to CRVO was treated by IVB. Three weeks after the first treatment, her visual acuity improved to $20 / 40$, CRT decreased to $244 \mu \mathrm{m}$ and MBR increased to 42.8 (Figure 2B). Four months after the first injection, she developed recurrent macular edema and her visual acuity once again decreased to 20/70. Examination revealed recurrent macular edema (CRT; $352 \mu \mathrm{m})$ and a decrease of MBR (23.2) (Figure 2C). Subsequently, the patient was given a second IVB that resulted in a decreased CRT $(284 \mu \mathrm{m})$ and an increased MBR (38.0) (Figure 2D), which was similar to that observed after her first IVB treatment. Two months after the second injection, CRT was scarcely changed $(289 \mu \mathrm{m})$ and there was only a slight decrease in MBR (30.7) (Figure 2E). Even though her CRT (477 $\mu \mathrm{m})$ increased and her visual acuity (20/70) did not further change, we noted a tendency for MBR to increase, with a value of 47.5 observed at five months after the second injection (Figure $2 \mathrm{~F}$ ). As a result, we decided to simply continue to observe the patient without additional treatment intervention. A graph of her 
Matsumoto et al. Ocular blood flow levels with CRVO after IVB.

MBR and CRT is shown in Figure 2G. Probably, she can keep retinal blood flow high in the future, and is considered to be a good prognosis.

Case Report 2. A 62-year-old man noticed a decrease in his right visual acuity and developed macular edema secondary to CRVO. Best-corrected visual acuity was 20/40 in his right eye. A color fundus photograph of the right eye with CRVO showed extensive retinal hemorrhage, tortuous retinal veins, and diffuse retinal edema (Figure 3A). Although a fluorescein angiogram of the same eye (Figure 3B) indicated dye staining of the vessel wall, neither a nonperfusion area nor neovascularization was found. At the time of the IVB treatment for macular edema secondary to CRVO, the patient's CRT was $475 \mu \mathrm{m}$ and MBR was 27.8 (Figure 4A). One month after the first IVB, the patient's visual acuity improved to 20/25, CRT decreased to $309 \mu \mathrm{m}$ and MBR increased to 37.5 (Figure 4B). Two months after his first injection, he developed recurrent macular edema and his visual acuity decreased to 20/30. Further examination revealed recurrent macular edema (CRT; $589 \mu \mathrm{m}$ ) and decrease of MBR (35.6) (Figure 4C). After receiving a second IVB, and CRT decreased (286 $\mu \mathrm{m})$, MBR increased (37.2), and his visual acuity improved to 20/20 (Figure 4D). However, two months after the second treatment, the macular edema resolved (CRT; $635 \mu \mathrm{m})$ and MBR decreased (31.3) (Figure 4E). Therefore, the patient was given a third IVB, which resulted in decreased CRT (313 $\mu \mathrm{m})$ and increased MBR (38.2) (Figure 4F). Two months after the third injection, he developed recurrent macular edema (573 $\mu \mathrm{m})$, his visual acuity decreased to 20/40, and MBR decreased to 27.8 (Figure 4G). Although the IVB treatments led to increases in MBR and decreases in CRT, MBR values tended to decline after each of the IVB administrations. A graph of 
Matsumoto et al. Ocular blood flow levels with CRVO after IVB.

his MBR and CRT is shown in Figure $4 \mathrm{H}$. His retinal blood flow may decrease in the future, and may result in ischemia type.

Case Report 3. A 43-year-old woman noticed a decrease in her right visual acuity and developed macular edema secondary to CRVO. Best-corrected visual acuity was $20 / 70$ in her right eye. A color fundus photograph of the right eye with CRVO showed extensive retinal hemorrhage, tortuous retinal veins, and soft exudates (Figure 5A). A fluorescein angiogram of the same eye (Figure 5B) indicated there was neither a nonperfusion area nor any neovascularization. Results from the OCT imaging showed that CRT of $413 \mu \mathrm{m}$ and MBR was 26.9 (Figure 6A). After undergoing IVB for macular edema secondary to CRVO, her visual acuity improved to $20 / 20$ at one month after the treatment. CRT decreased to $334 \mu \mathrm{m}$ and MBR increased to 36.6 (Figure 6B). However, within a one-year period, macular edema recurrence led to five additional IVBs being carried out in the patient. Her subsequent CRT and MBR measurements were $388 \mu \mathrm{m}$ and 43.2 (second IVB; Figure 6C), $348 \mu \mathrm{m}$ and 45.1 (third IVB; Figure 6D), $343 \mu \mathrm{m}$ and 48.6 (fourth IVB; Figure 6E), respectively. At one month after her fifth IVB, CRT was $346 \mu \mathrm{m}$ and MBR was 48.0 (Figure 6F), while at three months after her fifth IVB, CRT was $322 \mu \mathrm{m}$ and MBR was 48.6 (Figure 6G). After her fifth IVB treatment, she has had no further recurrence of macular edema, and her visual acuity has remained at 20/15. A graph of her MBR and CRT is shown in Figure $6 \mathrm{H}$. Probably, she can keep retinal blood flow high in the future, and is considered to be a good prognosis.

Discussion. We report three cases of macular edema secondary to non-ischemic CRVO in which the blood flow increased after IVBs. In case 1, 
Matsumoto et al. Ocular blood flow levels with CRVO after IVB.

CRT decreased and MBR increased after undergoing two IVBs. Based on the shape of the macular edema (Figure 2F) and the subsequent increase in blood flow, we do not believe the recurrence of macular edema was due to either recurrence or aggravation of CRVO condition. In this particular case, the improvement of macular edema and the blood flow may have been due to simply observing the natural course of the recovery after the second IVB treatment. In case 2, even though CRT decreased and MBR increased after each of the IVBs, MBR tended to fall when macular edema recurred. Since it is possible that the blood flow may continue to decrease, this might suggest that the patient may result in ischemic CRVO. As the patient's condition has yet to be stabilized, additional follow-ups will need to be done in order to find the best way to effect a cure. In case 3, even though CRT decreased and MBR increased after each of the IVB treatments, the patient was able to achieve and maintain good visual acuity, and her overall prognosis was good.

While the treatments were ultimately successful in cases 1 and 3, case 2 did not fare as well. These results suggest there may very well be a difference in the pathogenesis between case 2 and the other patients.

Although the pathogenesis of CRVO has yet to be fully understood, the major stimulus for the formation of macular edema and neovascularization in patients with CRVO appears to be hypoxia-induced production of VEGF, which is an angiogenic factor that promotes angiogenesis and increases permeability. ${ }^{1}$ Therefore, drugs that inhibit the bioactivity of VEGF represent a new paradigm for the treatment of both retinovascular disease and macular edema. A previous study has shown that enlargement of the foveal avascular zone and 
Matsumoto et al. Ocular blood flow levels with CRVO after IVB.

the intercapillary area causes localized retinal hypoxia. ${ }^{7}$ Subsequently, this localized macular ischemia accompanied by CRVO may then promote an increased production of cytokines such as VEGF. It also has been reported that VEGF-induced vascular permeability is mediated, in part, by intercellular adhesion molecule 1 (ICAM-1)-mediated retinal leukostasis. ${ }^{8}$ Leukostasis coincides with enhanced vascular permeability and capillary non-perfusion, which results in the over-expression of VEGF and a subsequent development of macular edema. In addition, Noma et al. reported finding a negative correlation between the perifoveal capillary blood-flow velocity and the retinal thickness at the central fovea in patients with branch retinal vein occlusion. ${ }^{9}$ Inhibition of VEGF would be expected to prevent or reduce ICAM-1, thereby secondarily improving blood flow. In some patients, however, bevacizumab therapy was not found to be effective, despite the administration of multiple intravitreal injections. ${ }^{10}$ Responses to IVB can differ from case to case, and thus, it may be that IVB is only effective in cases in which the VEGF concentration is high. Likewise, when elements other than VEGF participate, there may be no reaction to an IVB. Furthermore, these reactions may also be restricted due to bevacizumab's limited duration of action.

Even though the background for CRVO pathology can vary, LSFG may be useful in both determining the CRVO prognosis and in evaluating the treatment efficacy.

In the present report, we only observed a few cases over a short period of time and thus, a further study that examines a larger number of cases over a longer follow-up period will need to be undertaken. Moreover, in order to definitely 
Matsumoto et al. Ocular blood flow levels with CRVO after IVB.

confirm the present results, blood flows before and after IVB in CRVO cases will also need to be determined by other types of measurement devices. 
Matsumoto et al. Ocular blood flow levels with CRVO after IVB.

\section{References}

1. Aiello LP, Avery RL, Arrigg PG, et al. Vascular endothelial growth factor in ocular fluid of patients with diabetic retinopathy and other retinal disorders. N Engl J Med 1994; 331(22):1480-1487.

2. Noma $\mathrm{H}$, Funatsu $\mathrm{H}$, Yamasaki $\mathrm{M}$, et al. Pathogenesis of macular edema with branch retinal vein occlusion and intraocular levels of vascular endothelial growth factor and interleukin-6. Am J Ophthalmol 2005; 140(2):256-261.

3. Sugiyama T, Araie M, Riva CE, et al. Use of laser speckle flowgraphy in ocular blood flow research. Acta Ophthalmol 2010; 88(7):723-729.

4. Yaoeda K, Shirakashi M, Funaki S, et al. Measurement of microcirculation in the optic nerve head by laser speckle flowgraphy and scanning laser Doppler flowmetry. Am J Ophthalmol 2000; 129(6):734-739,

5. Aiello LP, Brucker AJ, Chang S, et al. Evolving guidelines for intravitreous injections. Retina 2004; 24(5 Suppl):S3-19.

6. Aizawa N, Yokoyama $\mathrm{Y}$, Chiba N, et al. Reproducibility of retinal circulation measurements obtained using laser speckle flowgraphy-NAVI in patients with glaucoma. Clin Ophthalmol 2011; (5):1171-1176.

7. Remky A, Wolf S, Knabben H, et al. Perifoveal capillary network in patients with acute central retinal vein occlusion. Ophthalmology 1997; 104(1):33-37.

8. Miyamoto K, Khosrof S, Bursell SE, et al. Vascular endothelial growth factor (VEGF)-induced retinal vascular permeability is mediated by intercellular adhesion molecule-1 (ICAM-1). Am J Pathol 2000; 156(5):1733-1739.

9. Noma $\mathrm{H}$, Funatsu $\mathrm{H}$, Sakata $\mathrm{K}$, et al. Macular microcirculation and macular oedema in branch retinal vein occlusion. Br J Ophthalmol 2009; 93(5):630-633.

10. Ach T, Hoeh AE, Schaal KB, et al. Predictive factors for changes in macular edema in intravitreal bevacizumab therapy of retinal vein occlusion. Graefes Arch Clin Exp Ophthalmol 2010; 248(2):155-159. 
Matsumoto et al. Ocular blood flow levels with CRVO after IVB.

\section{Figure legends}

Figure 1. Fundus photography (A) and fluorescein angiography (B) in case 1.

Fundus photograph shows extensive retinal hemorrhage, slightly tortuous retinal veins, and diffuse retinal edema (A). Fluorescein angiograph shows neither a nonperfusion area nor neovascularization (B).

Figure 2. Optical coherence tomography (OCT) image and color map produced using Laser Speckle Flowgraphy (LSFG) in case 1.

At the time of the patient's first visit, OCT imaging determined central retinal thickness (CRT) of $402 \mu \mathrm{m}$ and MBR was $32.8(\mathrm{~A})$. Three weeks after her first IVB, CRT decreased to $244 \mu \mathrm{m}$ and MBR increased to 42.8 (B). Four months later, her CRT increased to $352 \mu \mathrm{m}$ and MBR decreased to $23.2(\mathrm{C})$. After the second IVB, CRT decreased to $284 \mu \mathrm{m}$ and MBR increased to 38.0 (D), Two months after the second IVB, CRT was scarcely changed $(289 \mu \mathrm{m})$ and only a slight decrease in MBR (30.7) (E). with a further increase in both CRT (477 $\mu \mathrm{m})$ and MBR (47.5) noted at five months after the second injection (F). Course of CRT and MBR for case 1 is shown in the graph (G).

Figure 3. Fundus photography (A) and fluorescein angiography (B) in case 2. Fundus photograph shows extensive retinal hemorrhage, tortuous retinal veins, and diffuse retinal edema (A). Although the fluorescein angiograph shows dye staining of the vessel wall, neither a nonperfusion area nor neovascularization is observed (B).

Figure 4. Optical coherence tomography (OCT) image and color map produced using Laser Speckle Flowgraphy (LSFG) in case 2.

At the time of the patient's first visit, CRT was $475 \mu \mathrm{m}$ and MBR was $27.8(\mathrm{~A})$. 
Matsumoto et al. Ocular blood flow levels with CRVO after IVB.

One month after the first IVB, CRT decreased to $309 \mu \mathrm{m}$ and MBR increased to 37.5 (B). Two months after the first injection, CRT increased to $589 \mu \mathrm{m}$ and MBR decreased to 35.6 (C). After the second IVB, CRT decreased to $286 \mu \mathrm{m}$ and MBR increased to 37.2 (D), with the CRT then increasing to $635 \mu \mathrm{m}$ and the MBR decreasing to 31.3 at two months after the second IVB (E). After the third IVB, CRT decreased to $313 \mu \mathrm{m}$ and MBR increased to $38.2(\mathrm{~F})$, with CRT then increasing to $573 \mu \mathrm{m}$ and MBR decreasing to 27.8 at two months after the third injection (G). Course of CRT and MBR for case 2 is shown in the graph $(H)$.

Figure 5. Fundus photography (A) and fluorescein angiography (B) in case 3. Fundus photograph shows extensive retinal hemorrhage, tortuous retinal veins, and soft exudates (A). The fluorescein angiograph shows neither a nonperfusion area nor neovascularization (B).

Figure 6. Optical coherence tomography (OCT) image and color map produced using Laser Speckle Flowgraphy (LSFG) in case 3.

At the time of the patient's first visit, CRT was $413 \mu \mathrm{m}$ and MBR was $26.9(\mathrm{~A})$. One month after the first IVB, CRT decreased to $334 \mu \mathrm{m}$ and MBR increased to 36.6 (B). Her subsequent CRT and MBR measurements were $388 \mu \mathrm{m}$ and 43.2 (second IVB; (C)), $348 \mu \mathrm{m}$ and 45.1 (third IVB; (D)), $343 \mu \mathrm{m}$ and 48.6 (fourth IVB; (E)), respectively. At one month after her fifth IVB, CRT was $346 \mu \mathrm{m}$ and MBR was $48.0(F)$, while at three months after her fifth IVB, CRT was $322 \mu \mathrm{m}$ and MBR was $48.6(\mathrm{G})$. Course of CRT and MBR for case 3 is shown in the $\operatorname{graph}(\mathrm{H})$. 


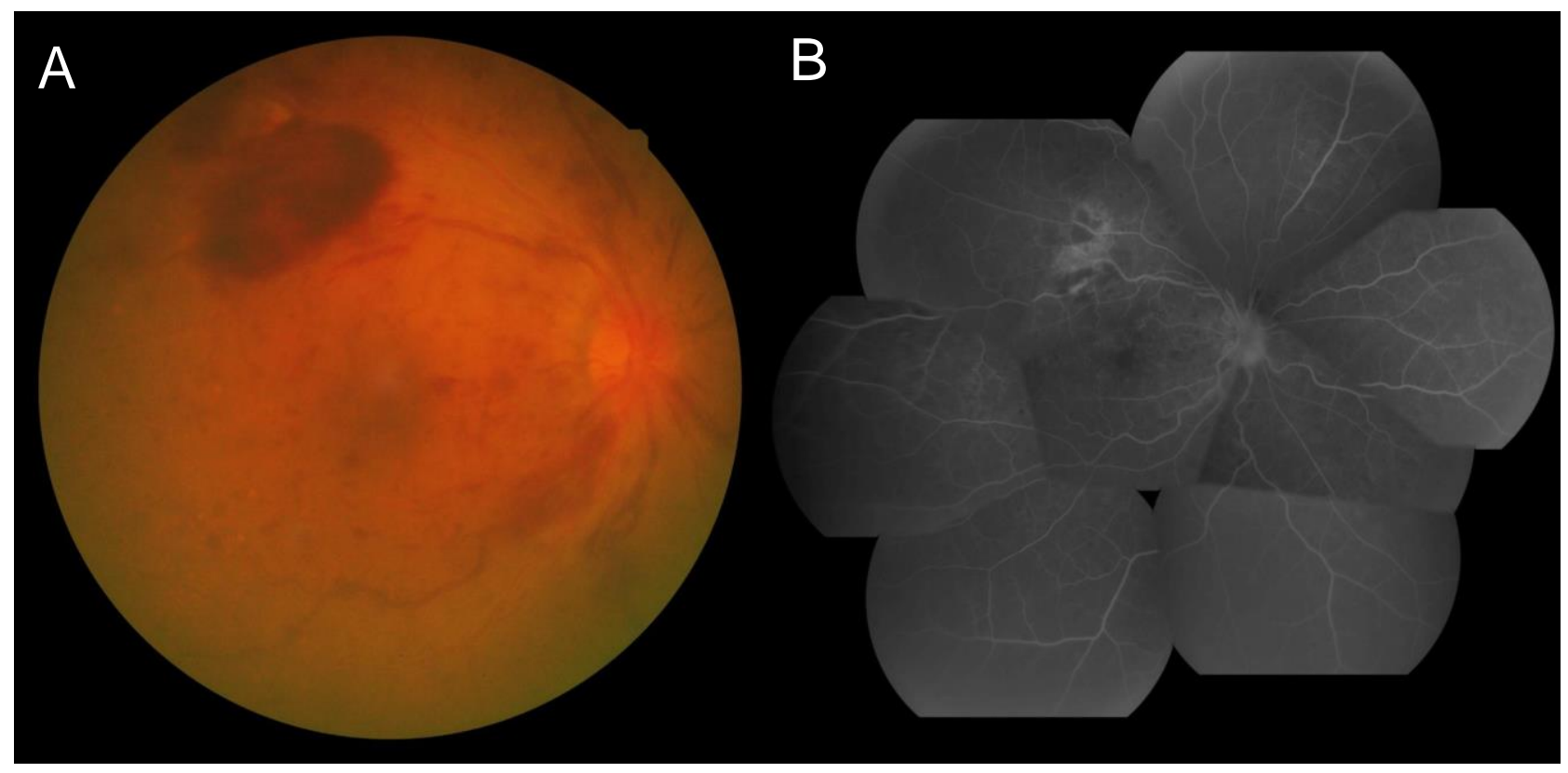

Figure 1 
A

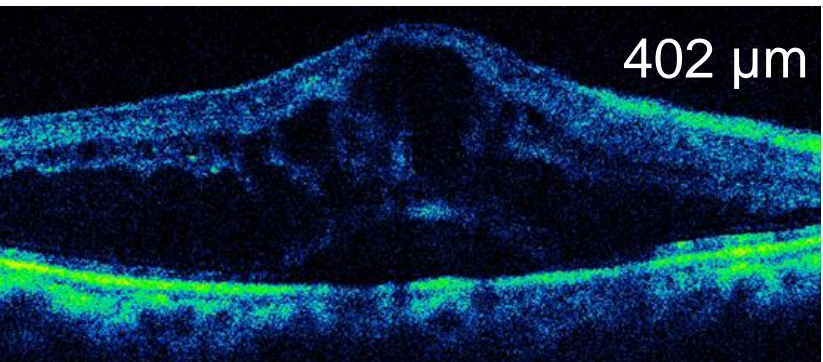

B

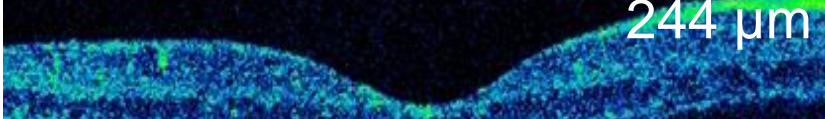
Q 20.0.2. H.

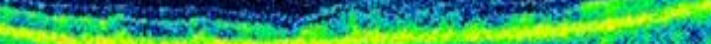

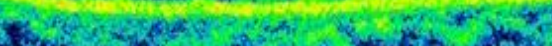

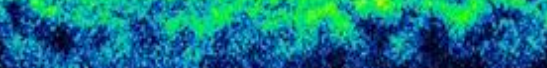

C

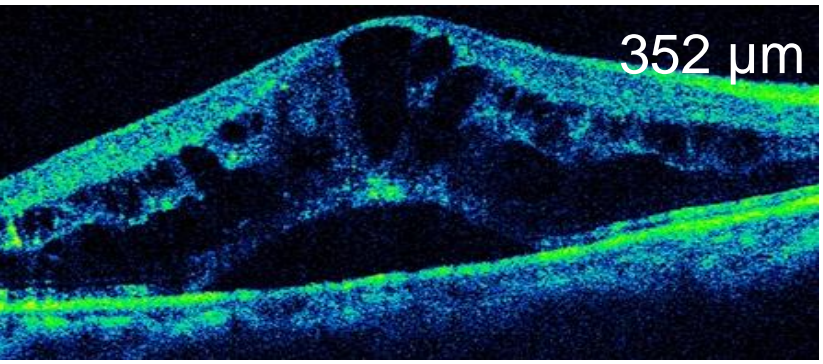

D

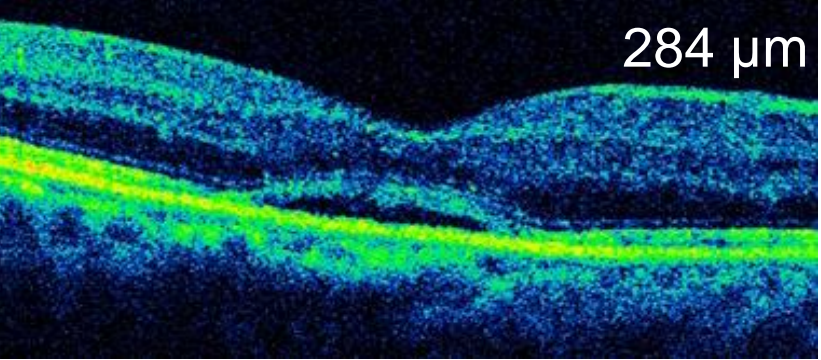

E

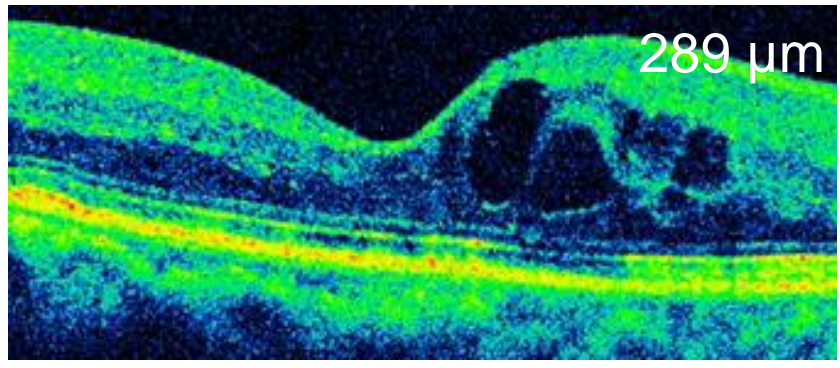

F

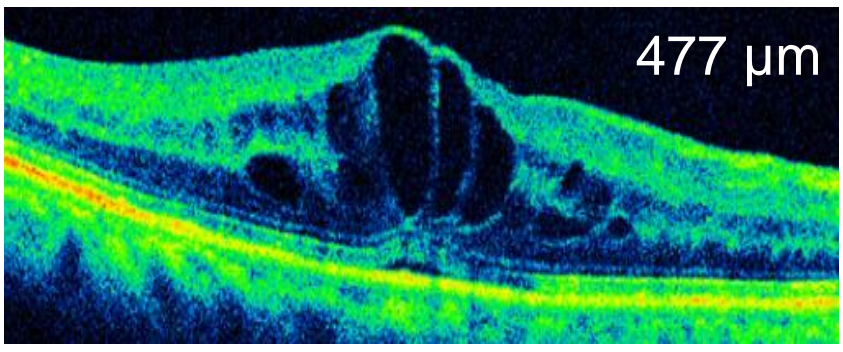

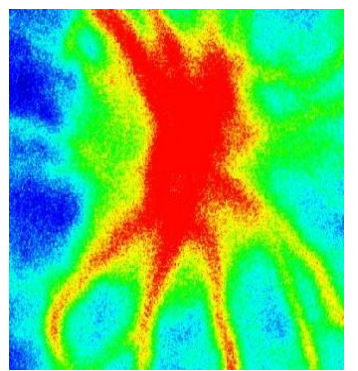

MBR

32.8

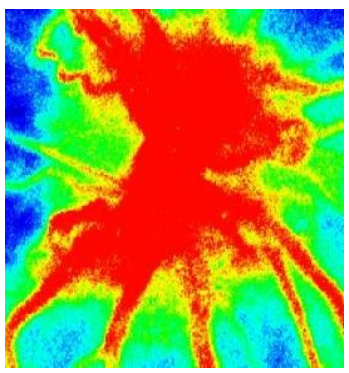

42.8

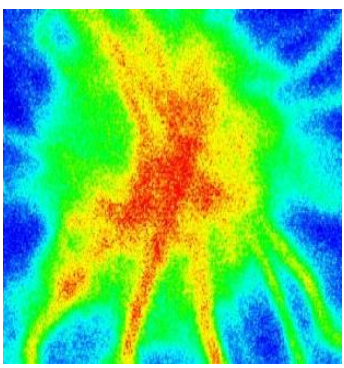

23.2

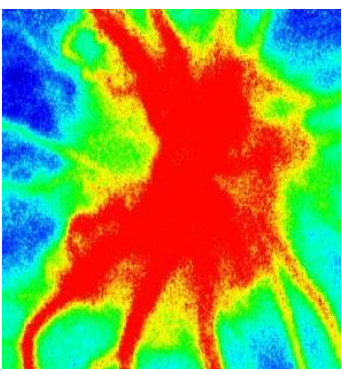

38.0

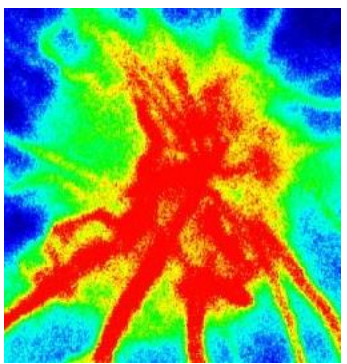

30.7

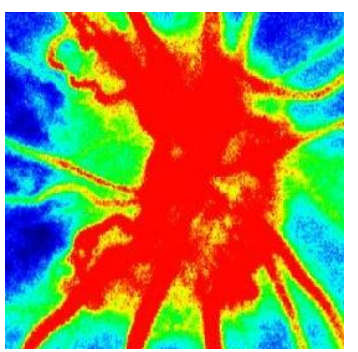

Figure 2 
G

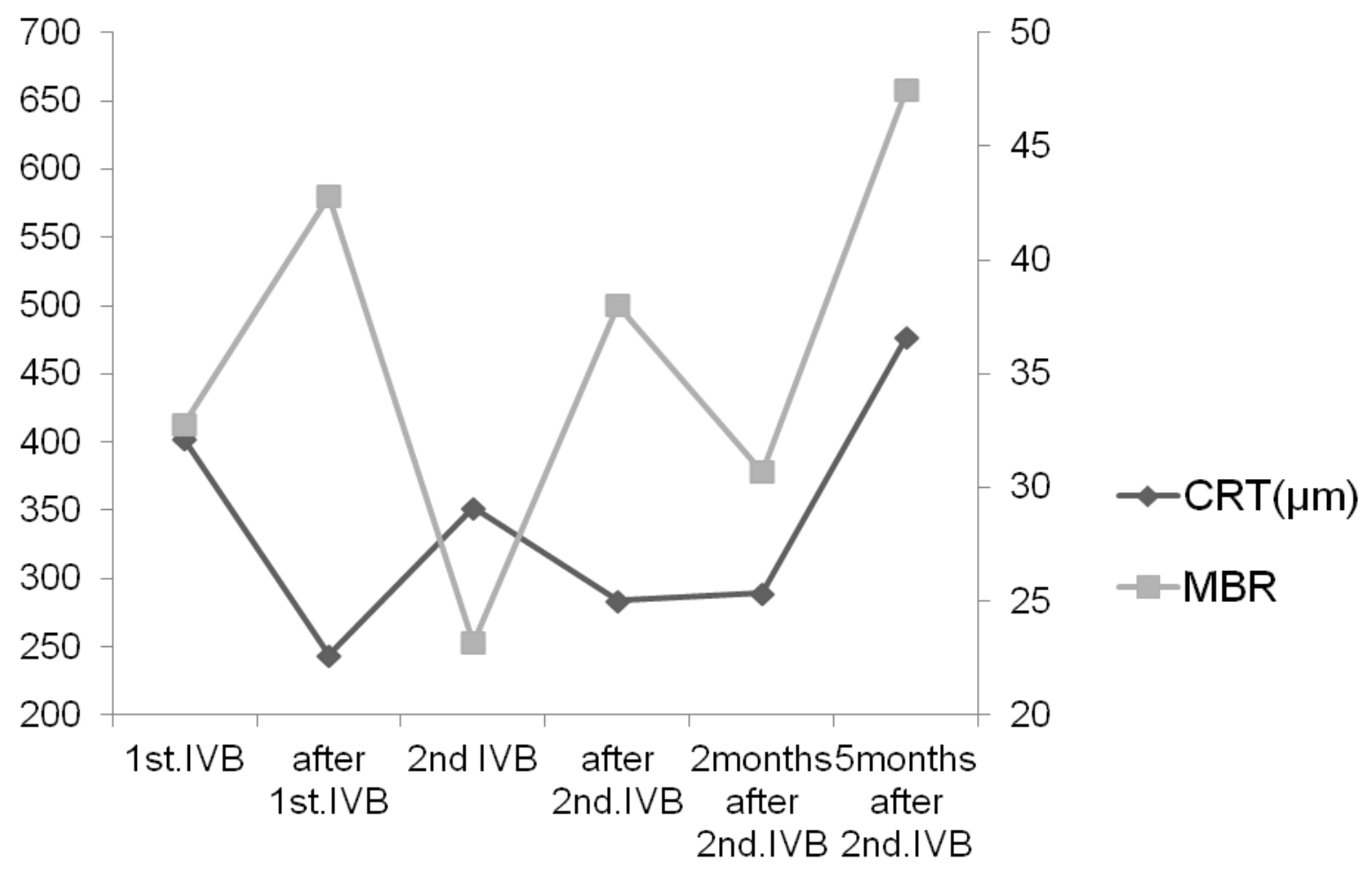

Figure 2 


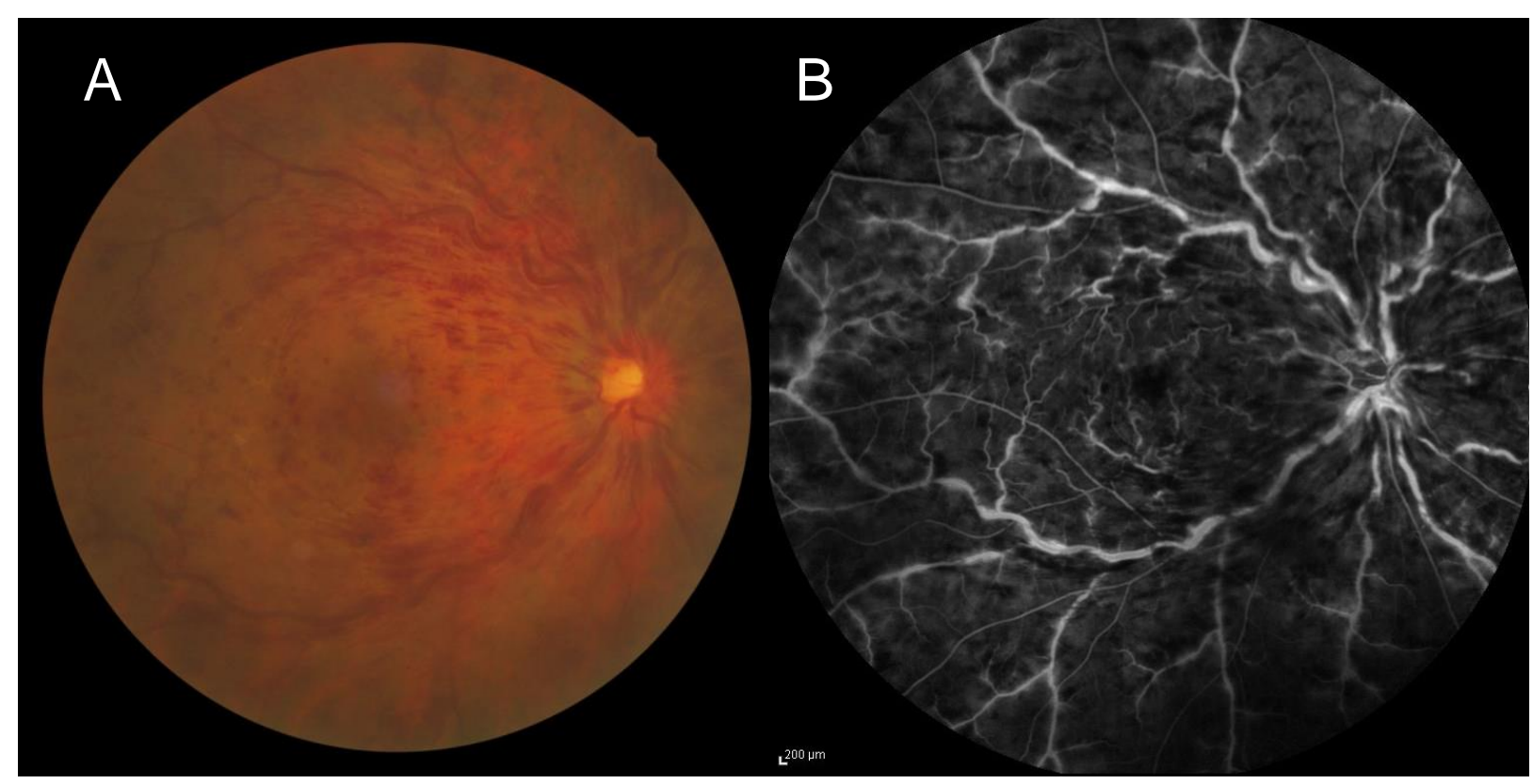

Figure 3 


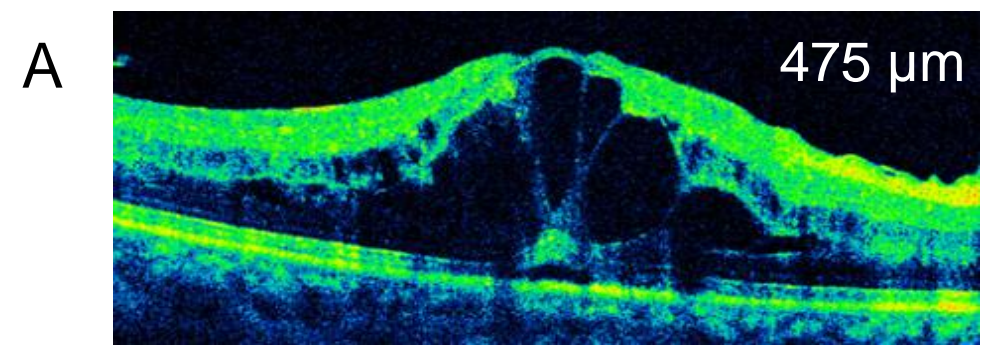

B

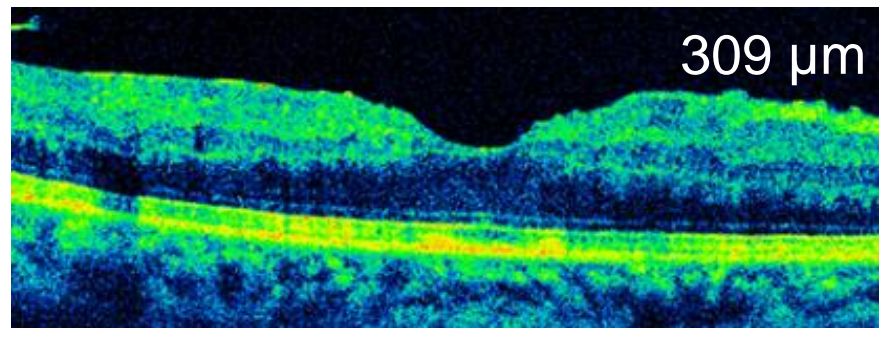

C

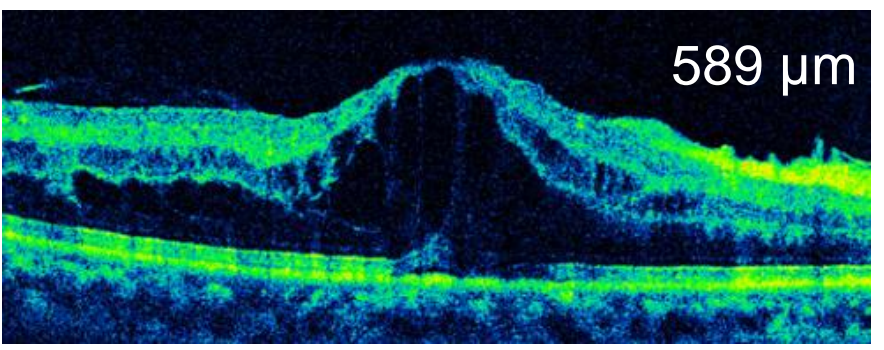

D

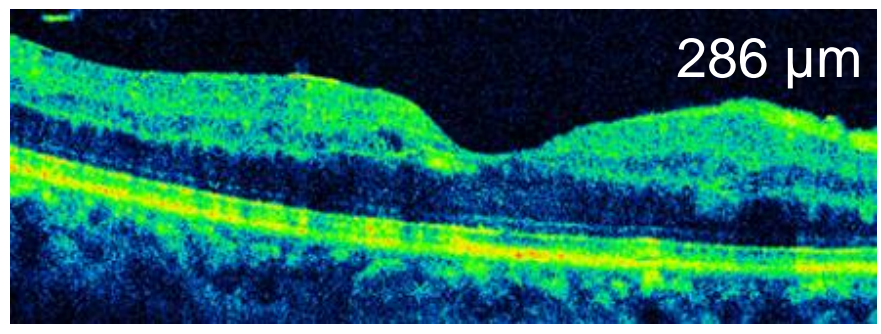

E

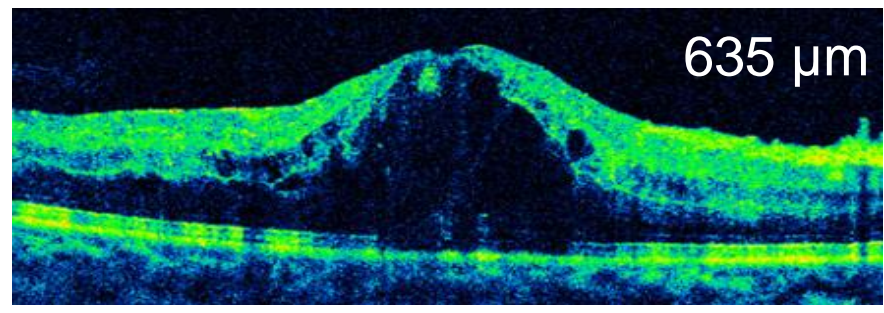

F

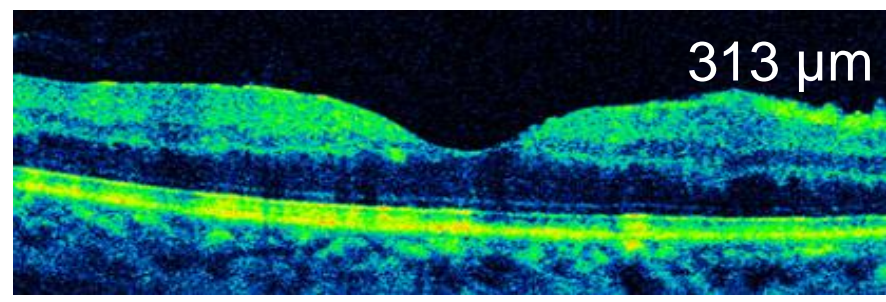

G

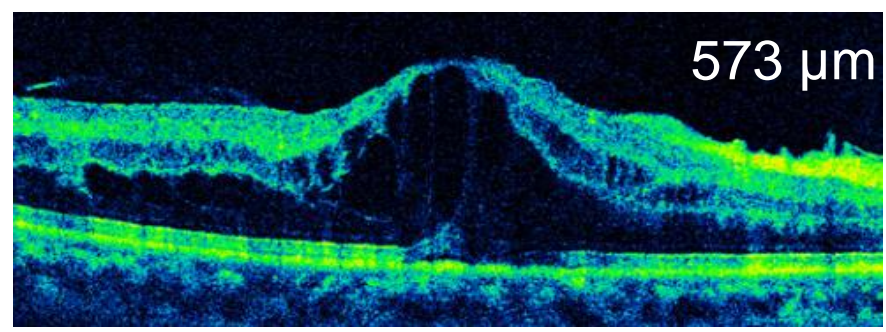

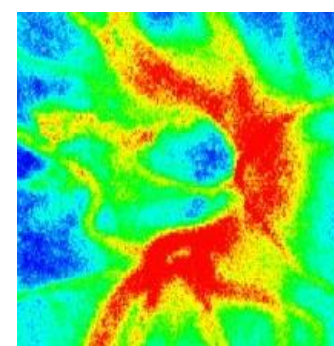

MBR

27.8

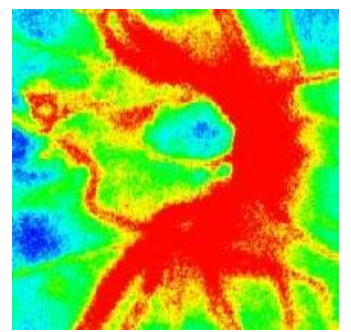

37.5

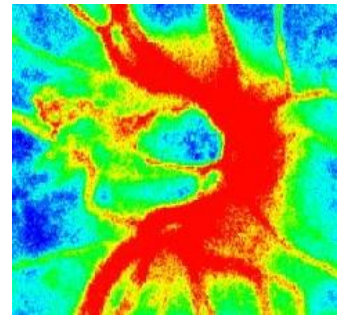

35.6

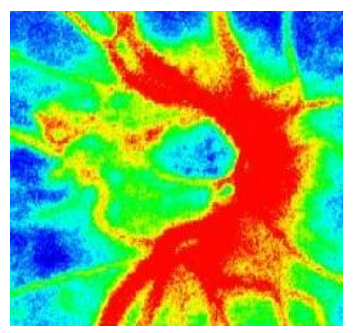

37.2

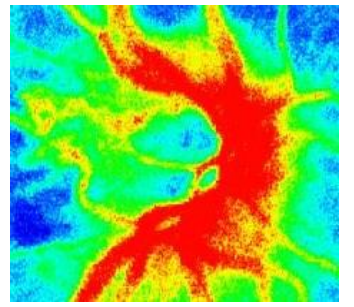

31.3

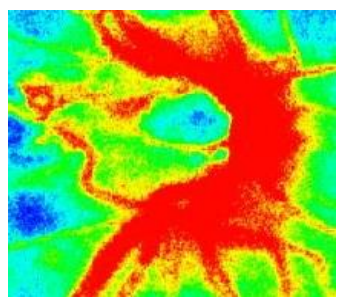

38.2

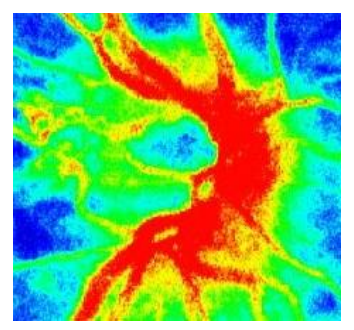

27.8 
$\mathrm{H}$

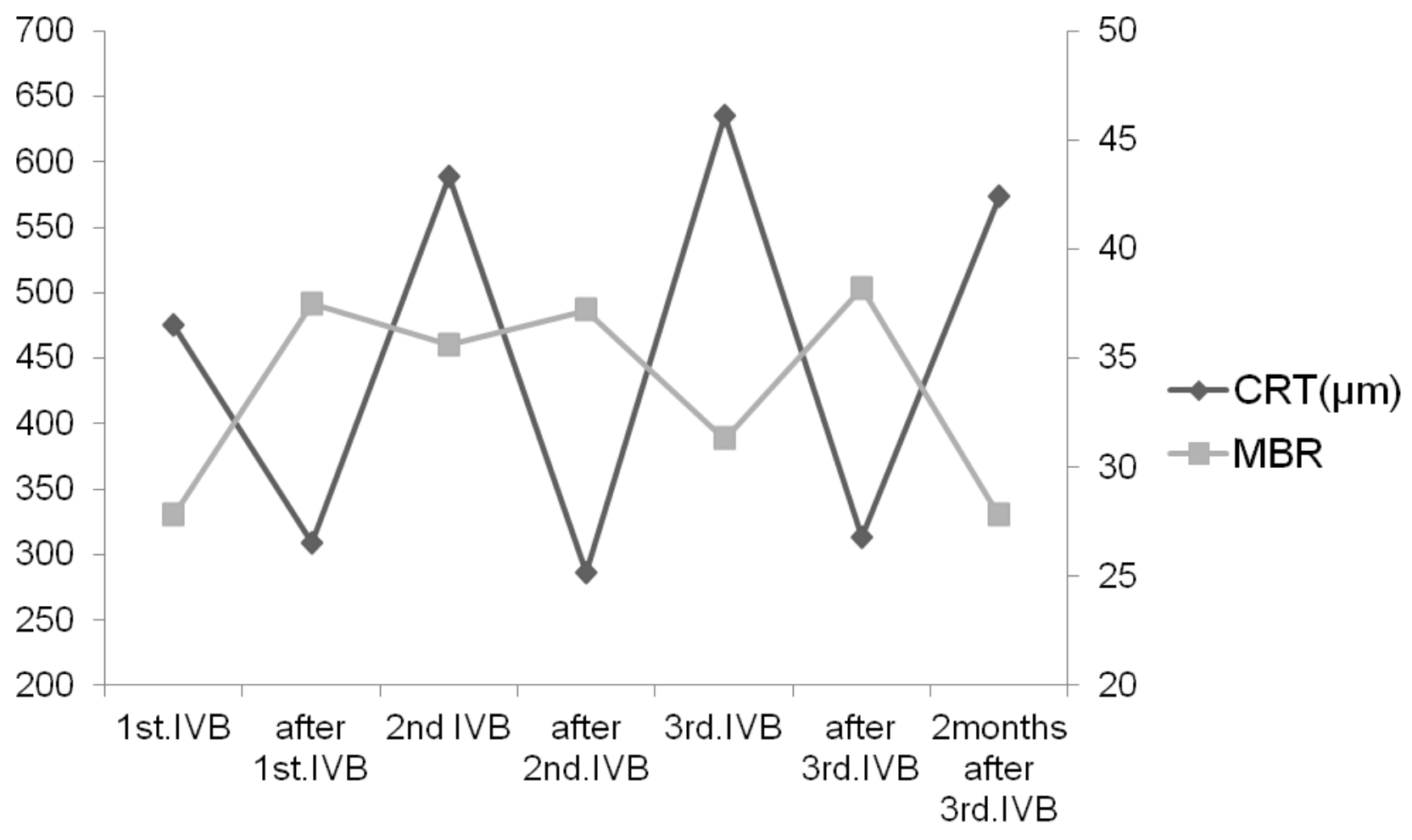

Figure 4 


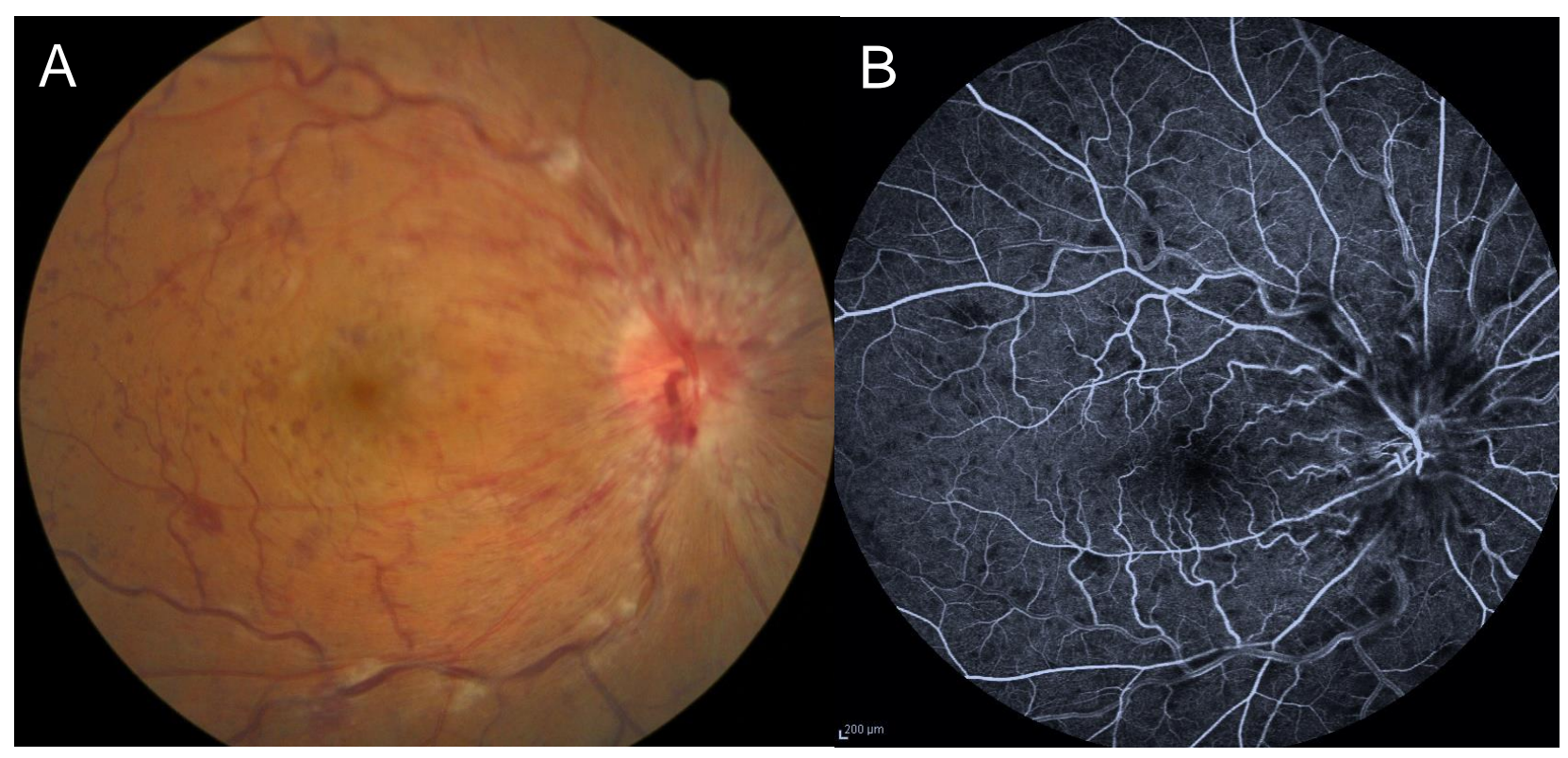

Figure 5 
A $413 \mu \mathrm{m}$

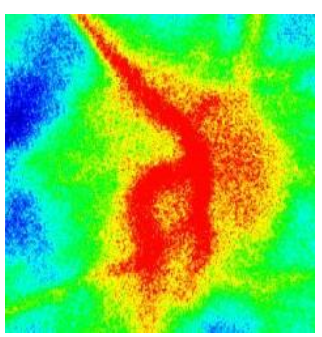

MBR 26.9

B $334 \mu \mathrm{m}$

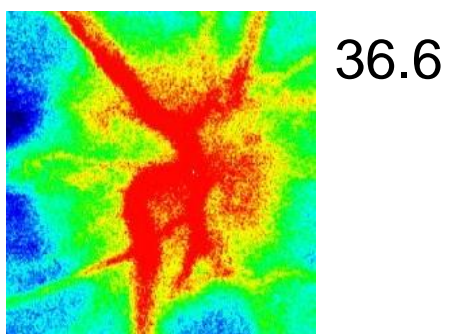

C $388 \mu \mathrm{m}$

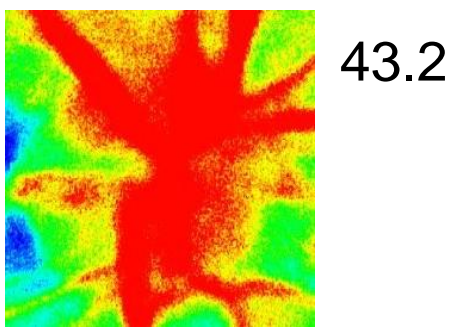

D $348 \mu \mathrm{m}$

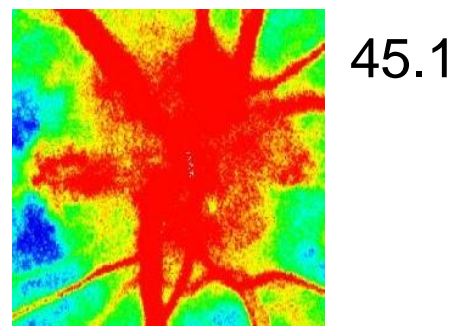

E 343.um

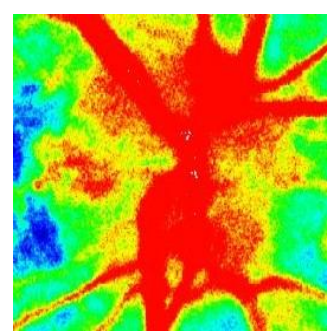

48.6

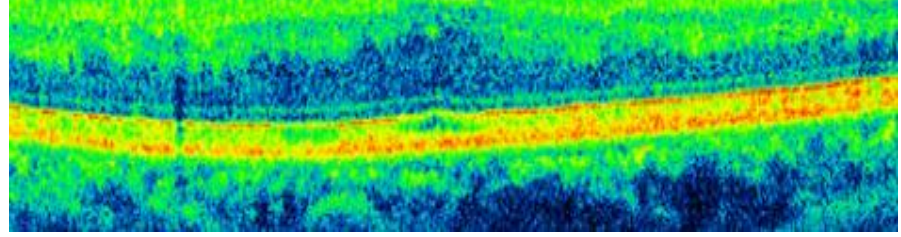

F $346 \mu \mathrm{m}$

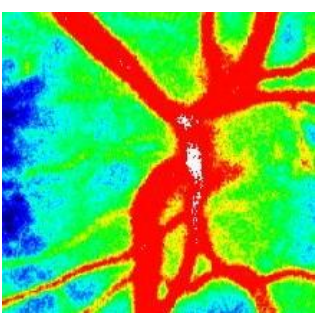

48.0

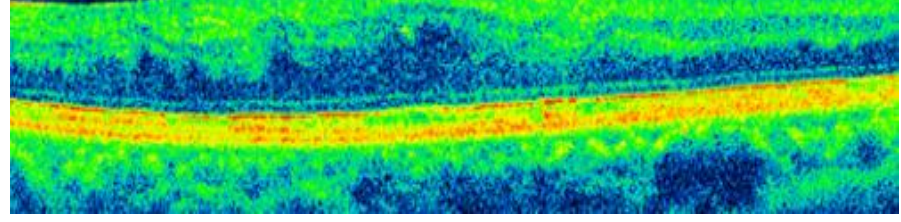

G $322 \mu m$

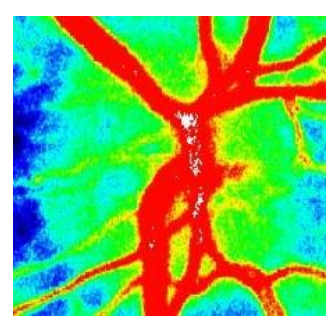

48.6

Figure 6 
$\mathrm{H}$

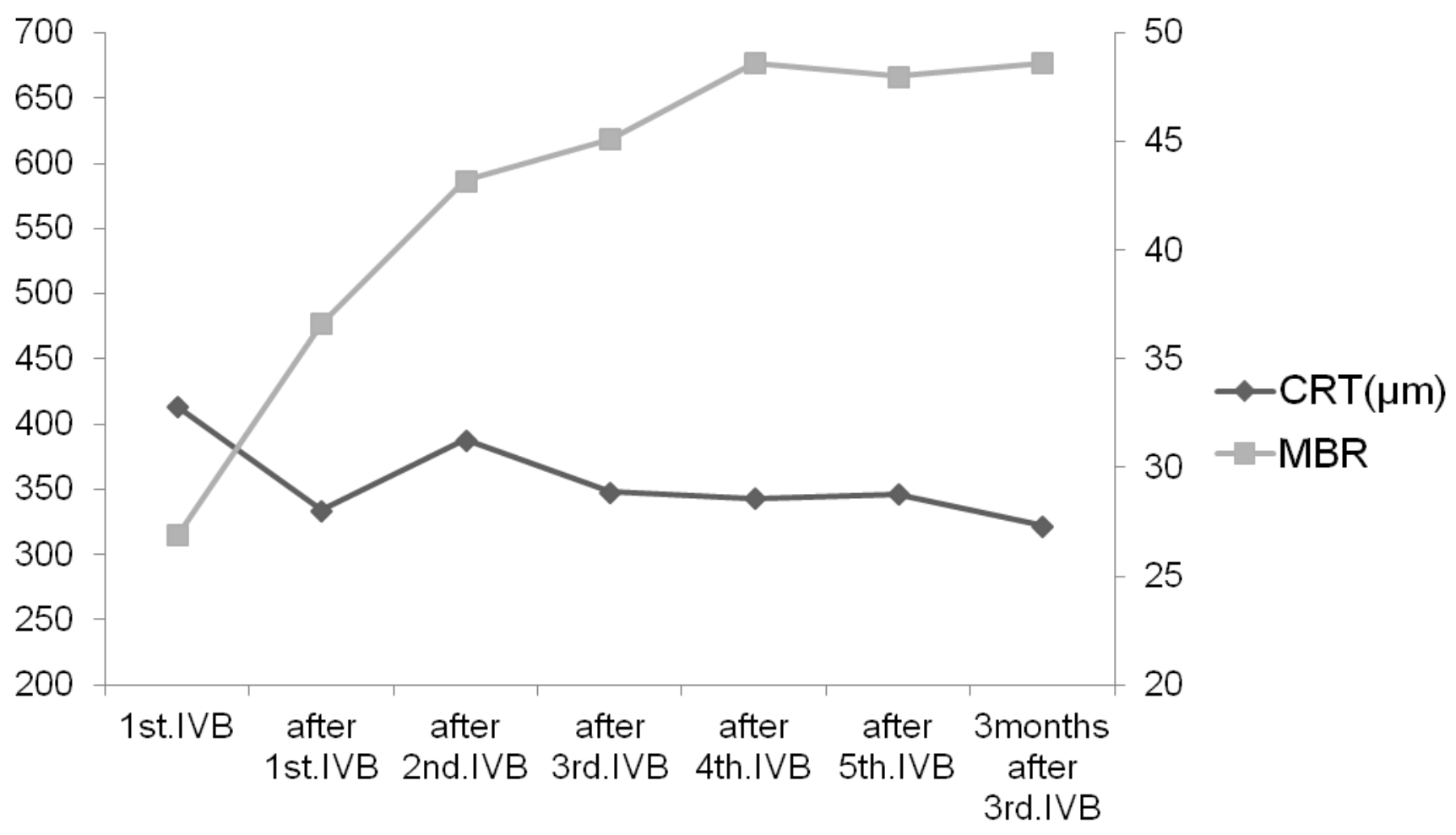

Figure 6 